\title{
Application of He's Method in Solving a System of Nonlinear Coupled Equations Arising in Non- Newtonian Fluid Mechanics
}

\author{
${ }^{1,3}$ A A Farooq, ${ }^{2}$ A M Siddiqui, ${ }^{3}$ M A Rana and ${ }^{4}$ T Haroon \\ ${ }^{1}$ COMSATS University of Science and Technology, Abbottabad, Pakistan \\ aliahmedfarooq@yahoo.com \\ ${ }^{2}$ Department of Mathematics, York Campus, Pennsylvania State University, USA \\ ${ }^{3}$ Department of Basic Sciences Riphah International University, Islamabad, \\ Pakistan \\ ${ }^{4}$ COMSATS University of Science and Technology, Islamabad, Pakistan
}

\begin{abstract}
In this paper, He's homotopy perturbation method is successfully employed to solve a nonlinear coupled system of ordinary differential equations that arises in the fully developed natural convection flow of a third grade fluid between two vertical parallel walls. The same problem is also solved by a numerical method (Runge-Kutta method of order 4) using the software MAPLE. The numerical results obtained by these methods are then compared and an excellent agreement is observed. This comparative study confirms the wide range applicability of HPM for nonlinear boundary value problems in different areas of science and engineering.
\end{abstract}

Keywords: Homotopy perturbation method, nonlinear boundary value problem, coupled equations, Natural convection flow, non-Newtonian fluid.

\section{Introduction}

The search for a comprehensive and easy to use tool for the solutions of nonlinear boundary value problems illuminating the flows of nonlinear fluids has been of great interest during the last decades. Various mathematical methods have been introduced for finding approximate solutions of nonlinear problems. One of the techniques that has become very popular recently is the homotopy perturbation method (HPM), proposed by He [1, 2]. The method introduces an effective and 
easy to use process for a wide variety of nonlinear equations, avoiding the restrictions and limitations of perturbation techniques. A vast number of nonlinear problems arising in the flows of non-Newtonian fluids in different geometries have recently been treated by this method; see for instance [3-7] and the references therein.

The objective of the present work is to extend homotopy perturbation method to solve a system of nonlinear equations that arises in the steady fully developed natural convection flow of a third grade fluid in a vertical channel. Recently, Ganji [8] have used adomian decomposition method to solve the same problem. However, an important advantage of HPM is that it avoids the difficulties arising in finding the adomian polynomials. HPM deforms a difficult problem into a set of problems which are easier to solve. In this method the solution is considered as the summation of an infinite series that usually converges rapidly to the exact solutions $[9,10]$. The analytical solutions for velocity and temperature obtained by HPM are shown to be in good agreement with the existing numerical solutions. This study clearly indicates that the proposed method may be considered as an alternative technique for solving a system of coupled nonlinear equations arising in non-Newtonian fluid flow problems.

\section{Governing equations}

We consider a steady, incompressible third order fluid between two vertical infinite parallel plates separated by a distance $2 b$. The plates at $x=-b$ and $x=+b$ are kept at constant temperatures $\Theta_{1}$ and $\Theta_{2}$, respectively, where $\Theta_{1}>\Theta_{2}$. The difference in temperatures causes the fluid near the plate at $x=-b$ to rise and the fluid near the plate at $x=+b$ to fall.

Rajagopal [11] has demonstrated that by using the similarity transformations

$$
v=\frac{V}{V_{0}}, \quad \eta=\frac{x}{b}, \quad \theta=\frac{\Theta-\Theta_{m}}{\Theta_{1}-\Theta_{2}},
$$

the momentum and energy equations can be reduced to the following system of nonlinear differential equations:

$$
\begin{aligned}
& \frac{d^{2} v}{d \eta^{2}}+6 \delta\left(\frac{d v}{d \eta}\right)^{2} \frac{d^{2} v}{d \eta^{2}}+\theta=0 \\
& \frac{d^{2} \theta}{d \eta^{2}}+E \cdot \operatorname{Pr}\left(\frac{d v}{d \eta}\right)^{2}+2 \delta E \cdot \operatorname{Pr}\left(\frac{d v}{d \eta}\right)^{4}=0,
\end{aligned}
$$

with $E=\frac{V_{0}^{2}}{c\left(\Theta_{1}-\Theta_{2}\right)}, \operatorname{Pr}=\frac{\mu c}{k}$ and $\delta=\frac{6 \beta_{3} V_{0}^{2}}{\mu b^{2}}$,

where $E$ is the Eckert number, Pr is the Prandtl number, $\delta$ is the non-Newtonian parameter and $c$ is the specific heat constant of the fluid. The appropriate nondimensional boundary conditions are 


$$
\left.\begin{array}{ll}
v=0, & \theta=0.5 \text { at } \eta=-1 \\
v=0, & \theta=-0.5 \text { at } \eta=1
\end{array}\right\}
$$

In what follows, we shall solve the system (2) and (3) using HPM. The system is coupled and highly nonlinear.

\section{Solution by HPM}

A detailed description of HPM is given in [1-4]. Here, we apply this method to the system (2) and (3). According to the HPM, we assume that the initial guess approximations subject to the boundary conditions (5) are

$$
v_{0}(\eta)=\frac{1}{12}\left(\eta^{3}-\eta\right), \quad \theta_{0}(\eta)=-\frac{1}{2} \eta
$$

and construct the following homotopies:

$$
\begin{aligned}
& (1-p)\left(v^{\prime \prime}-v_{0}^{\prime \prime}\right)+p\left[v^{\prime \prime}+6 \delta\left(v^{\prime}\right)^{2} v^{\prime \prime}+\theta\right]=0, \\
& (1-p)\left(\theta^{\prime \prime}-\theta_{0}^{\prime \prime}\right)+p\left[\theta^{\prime \prime}+E \cdot \operatorname{Pr}\left(v^{\prime}\right)^{2}+2 \delta E \cdot \operatorname{Pr}\left(v^{\prime}\right)^{4}\right]=0,
\end{aligned}
$$

Assume that the solutions of Eqs. (7) and (8) are of the following form

$$
\begin{aligned}
& v(\eta)=v_{0}+p v_{1}+p^{2} v_{2}+p^{3} v_{3}+\ldots \ldots, \\
& \theta(\eta)=\theta_{0}+p \theta_{1}+p^{2} \theta_{2}+p^{3} \theta_{3}+\ldots \ldots . .
\end{aligned}
$$

By substituting $v(\eta)$ and $\theta(\eta)$ from Eqs.(9) and (10) into (7) and (8) and after some simplification and rearrangement based on powers of $p$-terms, we have:

$$
\begin{aligned}
& p^{0}: \quad v_{0}^{\prime \prime}(\eta)=0, \quad \theta_{0}^{\prime \prime}(\eta)=0, \\
& v_{0}(-1)=0, v_{0}(1)=0, \quad \theta_{0}(-1)=0.5, \theta_{0}(1)=-0.5 \\
& p^{1}: \quad v_{1}^{\prime \prime}(\eta)=-\theta_{0}^{\prime \prime}-6 \delta\left(v_{0}^{\prime}\right)^{2} v_{0}^{\prime \prime}-\theta_{0}, \quad \theta_{1}^{\prime \prime}(\eta)=-\theta_{0}^{\prime \prime}-E \cdot \operatorname{Pr}\left(v_{0}^{\prime}\right)^{2}-2 \delta E \cdot \operatorname{Pr}\left(v_{0}^{\prime}\right)^{4} \text {, } \\
& v_{1}(-1)=0, v_{1}(1)=0, \quad \theta_{1}(-1)=0, \theta_{1}(1)=0 \\
& p^{2}: \quad v_{2}^{\prime \prime}(\eta)=-6 \delta\left(2 v_{0}^{\prime} v_{1}^{\prime} v_{0}^{\prime \prime}+\left(v_{0}^{\prime}\right)^{2} v_{1}^{\prime \prime}\right)-\theta_{1} \\
& \theta_{2}^{\prime \prime}(\eta)=-E \cdot \operatorname{Pr}\left(2 v_{0}^{\prime} v_{1}^{\prime}\right)-8 \delta E \cdot \operatorname{Pr}\left(v_{0}^{\prime}\right)^{3} v_{1}^{\prime}, \\
& v_{2}(-1)=0, v_{2}(1)=0, \quad \theta_{2}(-1)=0, \theta_{2}(1)=0
\end{aligned}
$$




$$
\begin{aligned}
& v_{3}^{\prime \prime}(\eta)=-6 \delta\left(\left(v_{1}^{\prime}\right)^{2} v_{0}^{\prime \prime}+2 v_{0}^{\prime} v_{2}^{\prime} v_{0}^{\prime \prime}+\left(v_{0}^{\prime}\right)^{2} v_{2}^{\prime \prime}+2 v_{0}^{\prime} v_{1}^{\prime} v_{1}^{\prime \prime}\right)-\theta_{2}, \\
& p^{3}: \\
& \theta_{3}^{\prime \prime}(\eta)=-E \cdot \operatorname{Pr}\left(2 v_{0}^{\prime} v_{2}^{\prime}+\left(v_{1}^{\prime}\right)^{2}\right)-2 \delta E \cdot \operatorname{Pr}\left(6\left(v_{0}^{\prime} v_{1}^{\prime}\right)^{2}+4\left(v_{0}^{\prime}\right)^{3} v_{2}^{\prime}\right), \\
& v_{3}(-1)=0, v_{3}(1)=0, \quad \theta_{3}(-1)=0, \theta_{3}(1)=0 \\
& v_{4}^{\prime \prime}(\eta)=-6 \delta\left(2 v_{0}^{\prime} v_{1}^{\prime} v_{2}^{\prime \prime}+2 v_{1}^{\prime} v_{2}^{\prime} v_{0}^{\prime \prime}+2 v_{0}^{\prime} v_{2}^{\prime} v_{1}^{\prime \prime}+\left(v_{1}^{\prime}\right)^{2} v_{1}^{\prime \prime}+\left(v_{0}^{\prime}\right)^{2} v_{3}^{\prime \prime}\right)-\theta_{3}, \\
& p^{4}: \\
& \theta_{4}^{\prime \prime}(\eta)=-E \cdot \operatorname{Pr}\left(2 v_{0}^{\prime} v_{3}^{\prime}+2 v_{0}^{\prime} v_{2}^{\prime}\right)-2 \delta E \cdot \operatorname{Pr}\left(4\left(v_{1}^{\prime}\right)^{3} v_{0}^{\prime}+4\left(v_{0}^{\prime}\right)^{3} v_{3}^{\prime}+12\left(v_{0}^{\prime}\right)^{2} v_{1}^{\prime} v_{2}^{\prime}\right) \text {, } \\
& v_{4}(-1)=0, v_{4}(1)=0, \quad \theta_{4}(-1)=0, \theta_{4}(1)=0
\end{aligned}
$$

Solving Eqs.(11)-(15) with boundary conditions, we have:

$$
\begin{aligned}
v_{0}(\eta) & =\frac{1}{12}\left(\eta^{3}-\eta\right), \quad \theta_{0}(\eta)=-\frac{1}{2} \eta \\
v_{1}(\eta) & =-\frac{\delta E \cdot \operatorname{Pr}}{48}\left(\frac{3 \eta^{7}}{14}-\frac{3 \eta^{5}}{10}+\frac{\eta^{3}}{6}-\frac{17 \eta}{210}\right) \\
\theta_{1}(\eta) & =-\frac{\delta E \cdot \operatorname{Pr}}{11520} \eta^{10}+\frac{\delta E \cdot \operatorname{Pr}}{5376} \eta^{8}-E \cdot \operatorname{Pr}\left(\frac{\delta}{5760}+\frac{1}{480}\right) \eta^{6}+E \cdot \operatorname{Pr}\left(\frac{\delta}{10368}+\frac{1}{288}\right) \eta^{4} \\
& -E \cdot \operatorname{Pr}\left(\frac{\delta}{20736}+\frac{1}{288}\right) \eta^{2}+E \cdot \operatorname{Pr}\left(\frac{\delta}{725760}+\frac{1}{480}\right) \\
v_{2}(\eta) & =\frac{\delta E \cdot \operatorname{Pr}}{1520640} \eta^{12}+\frac{3 \delta^{2}}{2816} \eta^{11}-\frac{\delta E \cdot \operatorname{Pr}}{483840} \eta^{10}-\frac{5 \delta^{2}}{2304} \eta^{9}+E \cdot \operatorname{Pr}\left(\frac{\delta}{322560}+\frac{1}{26880}\right) \eta^{8} \\
& +\frac{5 E \cdot \operatorname{Pr} \delta^{2}}{2688} \eta^{7}-E \cdot \operatorname{Pr}\left(\frac{\delta}{311040}+\frac{1}{8640}\right) \eta^{6}-\frac{61 E \cdot \operatorname{Pr} \delta^{2}}{67200} \eta^{5}+E \cdot \operatorname{Pr}\left(\frac{\delta}{248832}+\frac{1}{3456}\right) \eta^{4} \\
& +\frac{23 E \cdot \operatorname{Pr} \delta^{2}}{80640} \eta^{3}-E \cdot \operatorname{Pr}\left(\frac{19 \delta}{1451520}+\frac{1}{960}\right) \eta^{2}+E \cdot \operatorname{Pr}\left(\frac{29 \delta}{2737152}+\frac{67}{80640}\right) \\
\theta_{2}(\eta)= & \frac{\delta^{2}(E \cdot \operatorname{Pr})^{2}}{186368} \eta^{14}-\frac{\delta^{2}(E \cdot \operatorname{Pr})^{2}}{67584} \eta^{12}+\delta(E \cdot \operatorname{Pr})^{2}\left(\frac{\delta}{55296}+\frac{1}{5760}\right) \eta^{10}-\delta(E \cdot \operatorname{Pr})^{2}\left(\frac{179 \delta}{13547520}+\frac{1}{2688}\right) \eta^{8} \\
+ & \delta(E \cdot \operatorname{Pr})^{2}\left(\frac{191 \delta}{29030400}+\frac{1}{2880}\right) \eta^{6}-\delta(E \cdot \operatorname{Pr})^{2}\left(\frac{43 \delta}{17418240}+\frac{13}{60480}\right) \eta^{4} \\
+ & \delta(E \cdot \operatorname{Pr})^{2}\left(\frac{17 \delta}{17418240}+\frac{17}{120960}\right) \eta^{2}-\delta(E \cdot \operatorname{Pr})^{2}\left(\frac{46003 \delta}{87178291200}+\frac{1}{13440}\right)
\end{aligned}
$$

The other components $v_{i}(\eta)$ and $\theta_{i}(\eta)(i=3,4)$ are too long to be mentioned here; therefore, they are shown graphically. 


\section{Solution by Fourth-Order Runge-Kutta Method}

The best approximation for solving the system (2) and (3) that can be used is the Runge-Kutta method of order four. It is often used to solve differential equation systems. Ordinary differential equations of order four can be usually changed into third-order and then second-order equations and then to first order and then be solved through Runge-Kutta method.

\section{$5 \quad$ Skin Friction and Heat Transfer Rate}

The skin friction $S_{f}$ on the plate at $\eta=-1$ is directly proportional to $\frac{d v}{d \eta}$ and the heat transfer $H_{\theta}$ is directly proportional to $\frac{d \theta}{d \eta}$ and are given by

$$
\begin{gathered}
S_{f}(-1)=\frac{193 \delta^{2} \lambda}{138600}-\frac{6521 \delta \lambda}{748440}+\frac{\lambda}{756}+\frac{1}{6} \\
H_{\theta}(-1)=-\frac{967 \delta^{2} \lambda^{2}}{227026800}-\frac{\lambda^{2} \delta}{3780}+\frac{\delta \lambda}{7560}+\frac{\lambda}{180}-\frac{1}{2},
\end{gathered}
$$

where $\lambda=E . \operatorname{Pr}$.

\section{Concluding remarks}

In this paper, we have successfully employed HPM to solve the problem of fully developed laminar natural convection flow of a third grade fluid in a vertical channel. Explicit analytical expressions for velocity and temperature profiles have been obtained. This problem is also solved by a numerical method (the RungeKutta method of order 4) using the software MAPLE, and the numerical results are compared with those obtained by HPM in Table1. A close agreement between the two results is observed. Consequently, it is concluded that this method can be an alternative way for the solution of nonlinear coupled systems that have no exact analytic solutions. However, an important advantage of HPM is that it avoids the difficulties arising in finding the adomian polynomials.

Finally, some graphs are plotted to show the physical effects of the parameters $E$, $\operatorname{Pr}$ and $\delta$ on velocity and temperature profiles. Figs. 1 and 2 illustrate the results of $v(\eta)$ and $\theta(\eta)$ for various prandtl number $\operatorname{Pr}$ when $\delta=0.5$ and $E=1$. It is observed that by increasing $\operatorname{Pr}$ the velocity and temperature are increased respectively. By increasing $E$ in Fig. 3, the nondimensional velocity $v(\eta)$ is increased. Figs. 4 and 5 depict the effects of $\delta$ on the nondimensional velocity $v(\eta)$ and temperature $\theta(\eta)$ as well. 
It is also observed from Fig. 6 that an increase in non-Newtonian parameter $\delta$ keeping $\lambda$ fixed decreases the heat transfer linearly, which is becoming sharp with the increase in the values of $\lambda$, while the skin friction decreases parabolically up to a certain value of $\delta$ and after which it decreases. The skin friction is also decreased with an increase in $\lambda$.

Table1: Comparison of analytical solution with numerical solution when $\delta=0.5, E=\operatorname{Pr}=1$

\begin{tabular}{ccccc}
\hline$\eta$ & Analytic method & Numerical method & Analytic method & Numerical method \\
\hline & $v(\eta)$ & $v(\eta)$ & $\theta(\eta)$ & $\theta(\eta)$ \\
-1.0 & 0.0000000000 & 0.00000000 & 0.4999978227 & 0.50000000 \\
-0.8 & 0.02272441760 & 0.02305825 & 0.3987087198 & 0.40071781 \\
-0.6 & 0.03075402485 & 0.03135636 & 0.2991402382 & 0.30114634 \\
-0.4 & 0.02692219416 & 0.02771888 & 0.1995447363 & 0.20154660 \\
-0.2 & 0.01526309390 & 0.01617003 & 0.0998722471 & 0.10187244 \\
0.0 & -0.0001867384 & 0.00075121 & 0.00000000 & 0.00200104 \\
0.2 & -0.0115635750 & -0.01474168 & -0.100127747 & -0.09812529 \\
0.4 & -0.2728281391 & -0.02650959 & -0.200455132 & -0.19845291 \\
0.6 & -0.0310655200 & -0.003049585 & -0.300859154 & -0.29885930 \\
0.8 & -0.0237826390 & -0.02262939 & -0.401290764 & -0.399929217 \\
1.0 & 0.0000000000 & 0.00000000 & -0.500007059 & -0.5000000 \\
\hline
\end{tabular}




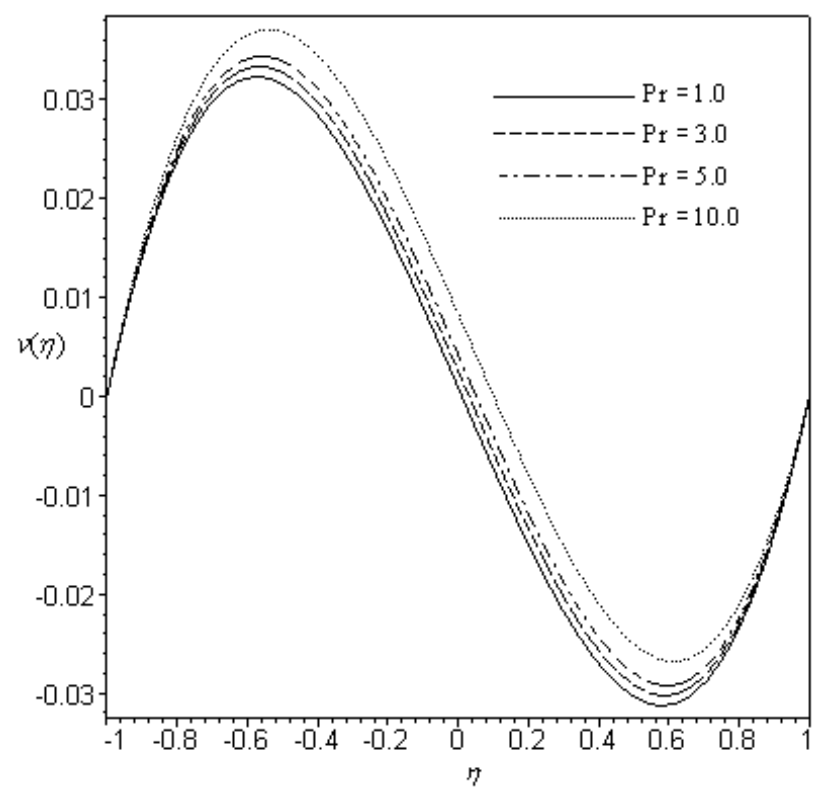

Fig.1 Results of $v(\eta)$ for various Pr when $E=1, \delta=0.5$

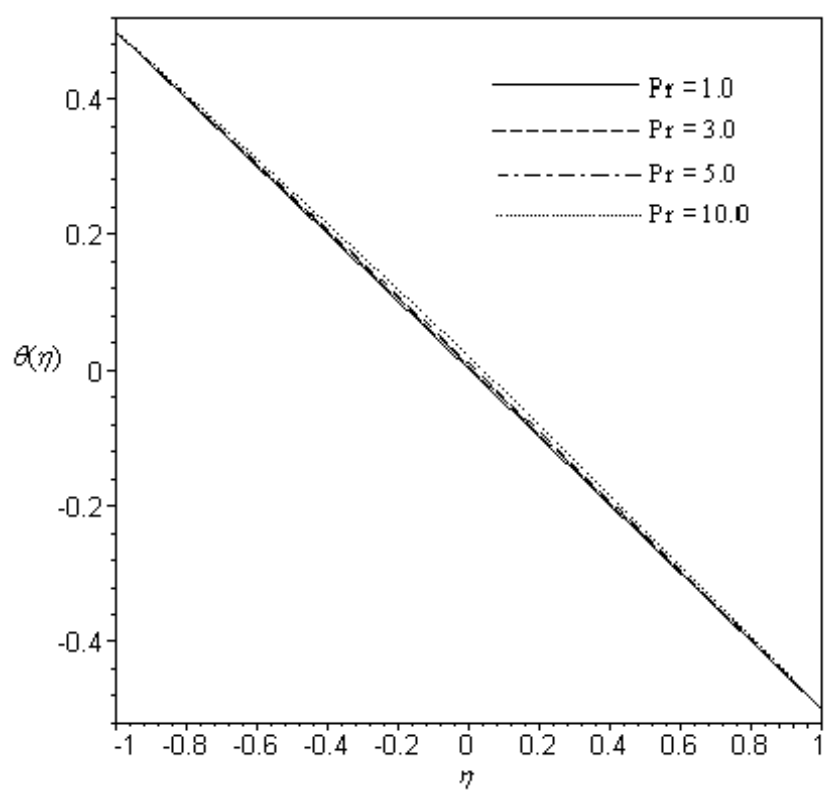

Fig.2 Results of $\theta(\eta)$ for various Pr when $E=1, \delta=0.5$ 


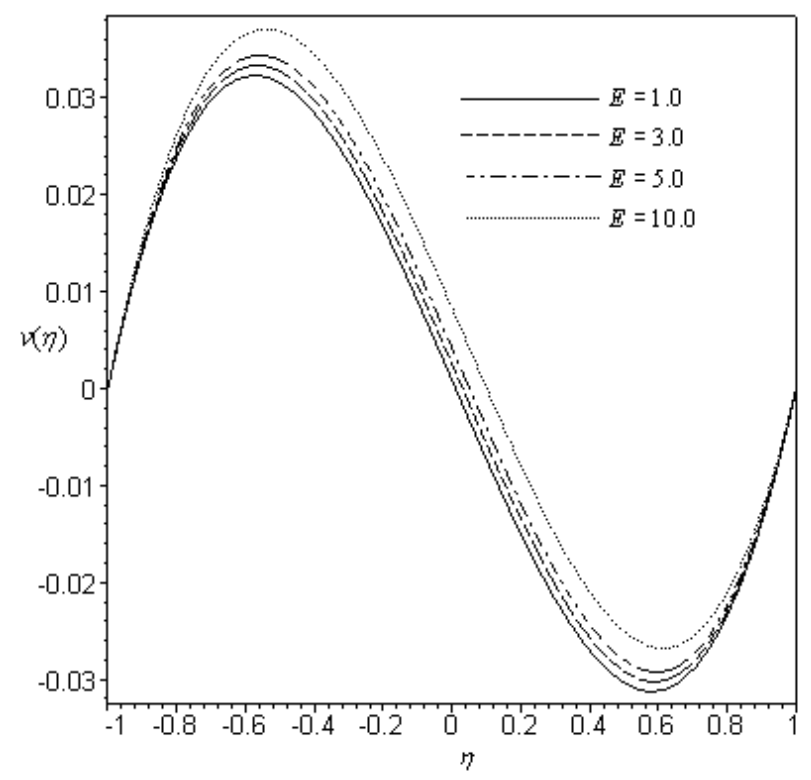

Fig.3 Results of $v(\eta)$ for various $E$ when $\operatorname{Pr}=1, \delta=0.5$

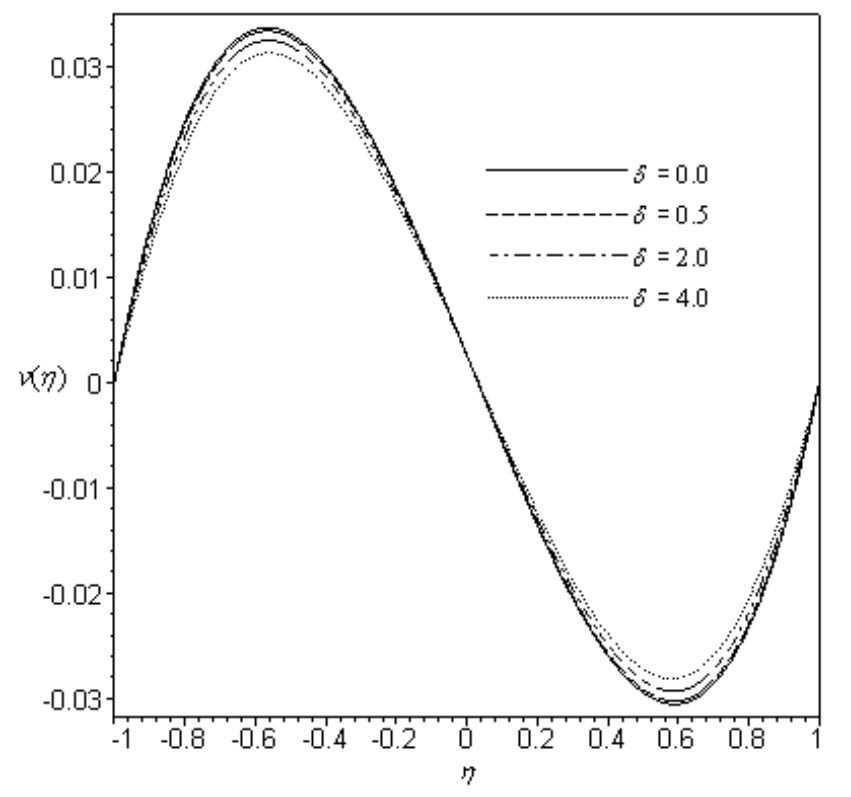

Fig.4 Results of $v(\eta)$ for various $\delta$ when $\operatorname{Pr}=3, E=3$ 


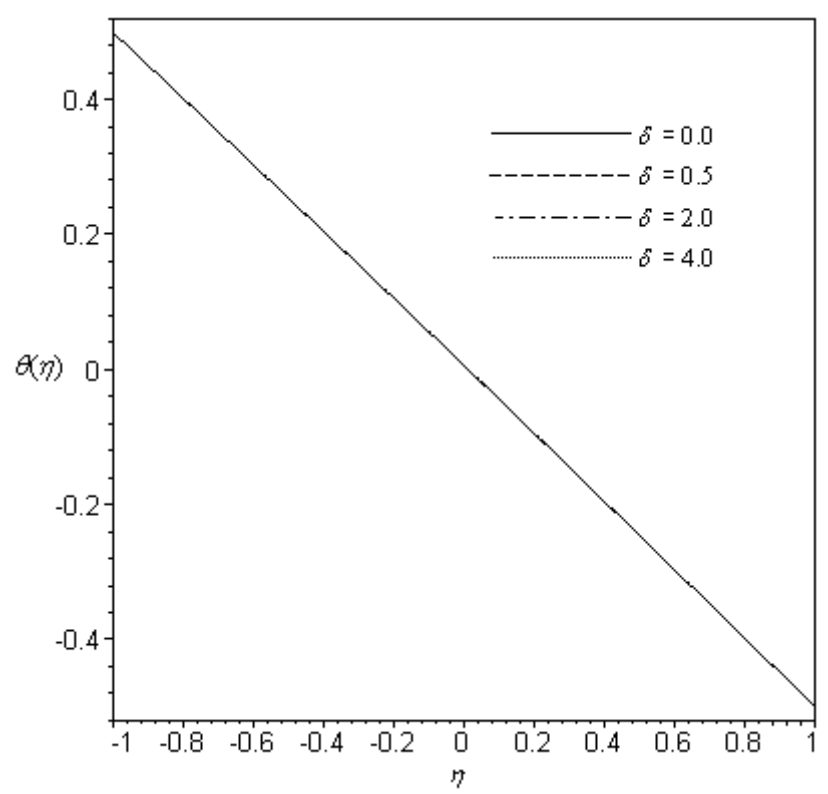

Fig.5 Results of $\theta(\eta)$ for various $\delta$ when $\operatorname{Pr}=3, E=3$ 

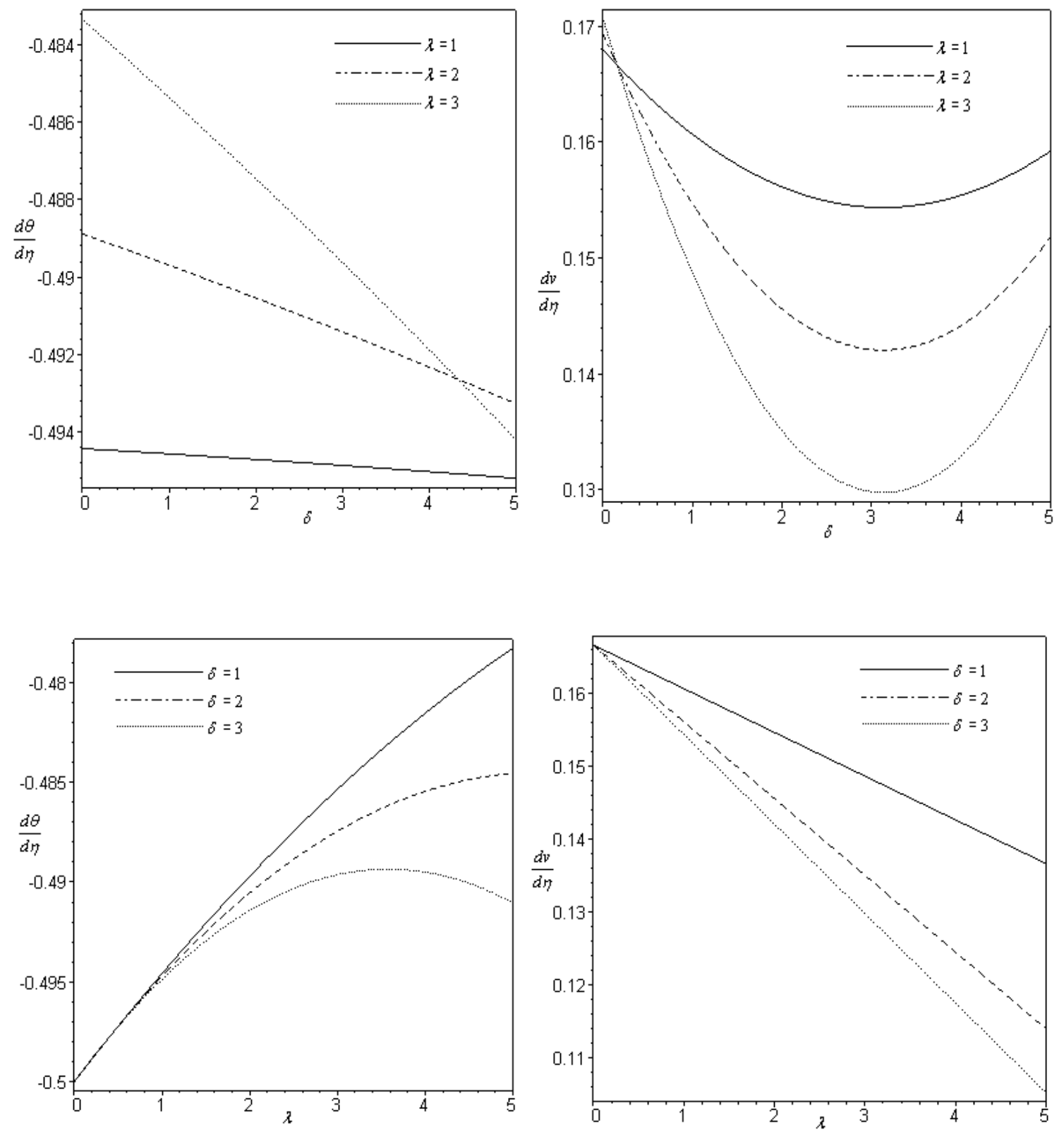

Fig.6 Variation of heat transfer $\frac{d \theta}{d \eta}(-1)$ and skin friction $\frac{d v}{d \eta}(-1)$ for different values of $\lambda$ and non-Newtonian parameter $\delta$ 


\section{References}

[1] J. H. He, A Review on Some New Recently Developed Nonlinear Analytical Techniques, Int. J. Nonlinear Sci. Numer. Simul.1(2000) 51-70

[2] J. H. He, Comparison of homotopy perturbation and homotopy analysis method, Applied mathematics and Computations 156 (2004) 527-539

[3] A. M. Siddiqui, R. Mahmod, Q. K. Ghori, Thin film flow of a third grade fluid on a moving belt by He's homotopy perturbation method, Int. J. Nonlinear Sci. Numer. Simul. 7 (2006)1-8

[4] J. H. He, Some asymptotic methods for strongly nonlinear equations, Internat. J. Modern Phys. B 20 (2006) 1141-1199

[5] Mustafa Inc and Ebru Cavlak, On numerical solutions of a new coupled MKdV system by using the Adomian decomposition method and He's Variational iteration method ,Physica Scripta 78(2008) 1-7.

[6] G. Adomian, Solving Frontier Problems of Physics: The Decomposition Method, Kluwer Academic Publishers, Boston, MA, (1994).

[7] A. M. Siddiqui, M. A. Hameed, B. M. Siddiqui, Q.K. Ghori, Use of Adomian decomposition method in the study of parallel plate flow of a third grade Fluid, Commun. Nonlinear Sci. Numer Simulat 15(2010)2388-2399.

[8] S. Abbasbanday, Numerical solutions of the integral equations: Homotopy perturbation method and Adomian's decomposition method, Applied Mathematics and Computation. 173 (2006)493-500.

[9] D.D.Ganji et., al., Analytic Solution of Natural Convection Flow of a NonNewtonian Fluid Between Two Vertical Flat Plates by Using Decomposition Method, Numerical Methods for Partial Differential Equations 27 (2010)1384-1395.

[10] M. Fathizadeh, F. Rashidi, Boundary layer convective heat transfer with pressure gradient using Homotopy Perturbation Method (HPM) over a flat plate, Chaos, Solitons and Fractals, 42 (2009)2413-2419.

[11] K.R. Rajagopal, T. Y. Na, Natural Convection flow of a non-Newtonian fluid between two vertical flat plates, Acta Mechanics 54 (1985) 239-46. 